\title{
Thymoma Type B3
}

National Cancer Institute

\section{Source}

National Cancer Institute. Thymoma Type B3. NCI Thesaurus. Code C7997.

Also known as well-differentiated thymic carcinoma, atypical thymoma, or epithelial thymoma, this type of thymoma displays morphologic characteristics of a welldifferentiated carcinoma. The majority of cases occur in the anterior mediastinum as Masaoka stage II or stage III tumors. It is almost always invasive, it recurs frequently, and metastasizes in approximately $20 \%$ of the cases. 